\title{
Timing dependence of peripheral pulse-wave-triggered pulsed arterial spin labeling.
}

\section{$\operatorname{AUTHOR}(S)$ :}

Fushimi, Yasutaka; Okada, Tomohisa; Yamamoto, Akira; Kanagaki, Mitsunori; Fujimoto, Koji; Togashi, Kaori

\section{CITATION:}

Fushimi, Yasutaka ...[et al]. Timing dependence of peripheral pulse-wave-triggered pulsed arterial spin labeling.. NMR in biomedicine 2013, 26(11): 1527-1533

\section{ISSUE DATE:}

2013-06-20

URL:

http://hdl.handle.net/2433/197928

\section{RIGHT:}

This is the peer reviewed version of the following article: Fushimi, Y., Okada, T., Yamamoto, A., Kanagaki, M., Fujimoto, K. and Togashi, K. (2013), Timing dependence of peripheral pulse-wave-triggered pulsed arterial spin labeling. NMR

Biomed., 26: 1527-1533, which has been published in final form at http://dx.doi.org/10.1002/nbm.2986. This article may be used for non-commercial purposes in accordance with Wiley Terms and Conditions for Self-Archiving.; This is not the published version. Please cite only the published version.; この論文は出版社版でありません。引用の際には出版社版

をご確認ご利用ください。 
Timing dependence of peripheral pulse-wave-triggered pulsed arterial spin labeling.

Running Title

Timing dependence of PPWT-ASL

Research Article 


\section{Abstract}

Arterial spin labeling (ASL) has been developed into a useful technique that is capable of noninvasively quantifying local cerebral blood flow (CBF) by using the water molecules in arterial blood as diffusible tracers. Pulsed ASL (PASL) is more strongly affected by the cardiac pulsation than Continuous ASL (CASL), because the tag bolus is shorter than the cardiac cycle in most cases. No reports have yet clarified the effects of multiple cardiac phases on the quantification of CBF in PASL when triggering is used. Fourteen subjects participated in this study. Peripheral pulse-wave-triggered (PPWT)-ASL was performed at various time-points at carotid artery (delay $0 \mathrm{~ms}$, 2nd point, foot, peak and tail) and compared with non-triggered (NT)-ASL. Regions of interest (ROIs) were applied based on anterior, middle and posterior cerebral artery (ACA, MCA, PCA) territories and CBFs were compared among different time-points and ROIs. PPWT-ASL strongly affects CBF values compared with NT-ASL in ACA and MCA territories, especially when measured at the foot of the carotid artery flow phase. CBF_NT was assumed to be approximately between minimum and maximum CBF with clear statistical significance in several ROIs at several time points of PPWT-ASL, and CBF_NT was assumed to resemble “randomly triggered” PPWT-ASL. In conclusion, PPWT-ASL strongly affects CBF 
values compared with NT-ASL, particularly with foot of carotid artery flow in ACA and MCA territories.

Abbreviations used: ACA, anterior cerebral artery; MCA, middle cerebral artery; PCA, posterior cerebral artery; PPWT, peripheral pulse-wave-triggered; ASL, arterial spin labeling; NT, non-triggered; PET, positron emission tomography; SPECT, single photon emission computed tomography; VFR, volumetric flow rate; MPRAGE, magnetization-prepared rapid gradient echo; PR, pulse rate; Q2TIPS, QUIPSSII with Thin-Slice TI1 periodic Saturation; FWHM, full-width at half-maximum; ANOVA, analysis of variance; SNR, signal to noise ratio; CV, Coefficients of variance; ROI, regions of interest. 
Text

\section{Introduction}

Arterial spin labeling (ASL) has been developed into a useful technique that is capable of noninvasively quantifying local cerebral blood flow (CBF) with good spatial and temporal resolution compared with other imaging modalities, such as positron emission tomography (PET), single photon emission computed tomography (SPECT), CT perfusion, and magnetic resonance (MR) perfusion (1-5). ASL uses the water molecules in arterial blood as diffusible tracers that are magnetically tagged by radiofrequency pulses.

ASL can be roughly categorized into pulsed ASL (PASL) $(2,3,6,7)$ and continuous ASL (CASL) $(1,8,9)$. Several novel ASL methods have recently been developed and introduced to research and clinical examinations, such as pseudocontinuous CASL (PCASL) (10), Look-Locker ASL (11), and 3D GRASE ASL (12). In most ASL methods, tagged blood is assumed to be delivered from the tagging region to the imaging region via simple plug flow. The magnetized, tagged blood eventually perfuses the target tissue, and tagged water spins exchange with brain parenchyma water spins in the capillary bed.

Flow in the carotid arteries is variable in the living body (13), showing 
characteristics of plug, laminar, and turbulent flow. Studies of volumetric flow rate (VFR) waveforms in the carotid arteries have exhibited time-varying flow velocity reflecting cardiac phases $(14,15)$. Dispersion of a bolus of blood in transit from the neck or cerebral arteries to the brain occurs due to the cardiac phase: transit delay of tagged blood is shorter in the cardiac systolic phase and longer in the diastolic phase according to the findings of a study of the two representative phases (16). A previous study showed the transit delay in PASL is more strongly affected by the cardiac pulsation than CASL, because the tag bolus is shorter than the cardiac cycle in most cases (17). No reports have yet clarified the effects of multiple cardiac phases on the quantification of CBF in PASL when triggering is used.

The present study conducted PASL with multiple cardiac phases using peripheral pulse gating and evaluated the effects of cardiac phases on the quantification of CBF. We compared peripheral pulse-wave-triggered (PPWT) ASL with non-triggered (NT) ASL.

\section{PATIENTS AND METHODS}

All study protocols were approved by the local institutional review board and written informed consent was obtained from all subjects. Participants comprised 14 
healthy volunteers (5 men, 9 women; mean age, $31.6 \pm 5.1$ years).

\section{Imaging protocol}

All subjects underwent MR imaging using a 3-T system (Magnetom Verio; Siemens, Erlagen, Germany) with a 12-channel phased-array head coil. A pulse oximeter provided by the MRI vendor was set on the left finger to provide a triggering signal at the peak of the pulse wave. First, a magnetization-prepared rapid gradient echo (MPRAGE) sequence was used to provide 3-dimensional (3D) anatomical images. Phase contrast imaging was conducted before ASL. The imaging plane was perpendicular to the common carotid artery, located $15 \mathrm{~mm}$ below the carotid bifurcation. The pulse wave in the common carotid artery was analyzed immediately using the software included in the MR scanner and each of the following time points of the wave visualized in Figure 1 were identified: delay $0 \mathrm{~ms}$; 2nd point (midpoint between delay $0 \mathrm{~ms}$ and foot); foot; peak; and tail. Note that we arbitrarily named the end of the reflux wave on the carotid pulse wave as the "tail". PPWT-ASL was performed for each time point. Tagging was synchronized with the start with the trigger of each peripheral pulse wave. The pulse wave information was simultaneously transmitted to the MRI system. Phase-contrast imaging was also conducted after ASL 
measurements to clarify the differences in time points of carotid artery flow timing compared with pre-ASL measurements.

NT-ASL and PPWT-ASLs with the aforementioned delay times were conducted for all subjects. Phase-contrast imaging was performed again after ASL using the same conditions as phase-contrast imaging before ASL.

\section{Imaging parameters}

MPRAGE was obtained as anatomical images under the following conditions: repetition time, $1700 \mathrm{~ms}$; echo time, $2.49 \mathrm{~ms}$; flip angle, 9; inversion time, $900 \mathrm{~ms}$; field of view, $250 \times 250 \mathrm{~mm}$; matrix, $0.98 \times 0.98 \mathrm{~mm}$; slice thickness, $1 \mathrm{~mm}$; and bandwidth, $170 \mathrm{~Hz} /$ pixel.

Scan parameters of phase-contrast imaging were as follows: repetition time, $16.75 \mathrm{~ms}$; echo time, $4.83 \mathrm{~ms}$; flip angle, 15; field of view, $300 \times 225 \mathrm{~mm}$; matrix, $1.17 \times 1.17 \mathrm{~mm}$; slice thickness, $8.0 \mathrm{~mm}$; and bandwidth, $300 \mathrm{~Hz} /$ pixel. One-directional foot-head through-plane phase-contrast MR imaging was performed. Velocity encoding was set to $100 \mathrm{~cm} / \mathrm{s}$ and retrospective gating with 128 phases applied during one average cardiac cycle resulted in a temporal resolution of 5-10 ms, depending on pulse rate (PR). 
NT-ASL and PPWT-ASL were performed using the second version of the QUIPSSII with Thin-Slice TI1 periodic Saturation (Q2TIPS) (6). Imaging parameters were as follows: repetition time, $3000 \mathrm{~ms}$; echo time, $14 \mathrm{~ms}$; field of view, $256 \times 256$ mm; matrix, $64 \times$ 64; slice thickness, 6 mm; interslice gap, 1.5 mm; 13 slices; partial Fourier acquisition rate, 7/8; TI1, 700 ms; TI1s, 1400 ms; TI2, 1600 ms; 45 pairs of labeled and unlabeled images; and scan times, 4 min 12 ms for NT-ASL, and slightly longer for PPWT-ASL. For quantitation, the following values were used: brain/blood partition coefficient of water $(\lambda), 0.9 \mathrm{~g} / \mathrm{mL}$; inversion efficiency $(\alpha), 0.95$; and longitudinal relaxation time of arterial blood (T1a), 1664 ms (18-23).

\section{Post-imaging analysis}

Unsubtracted magnitude images were realigned to the first image of the series for motion correction using a six-parameter rigid-body spatial transformation and coregistered to the corresponding MPRAGE images using MATLAB 7.0 software (Mathworks, Natick, MA, USA) with SPM5 statistical parametric mapping software (http://www.fil.ion.ucl.ac.uk/spm/). Control and labeled images were coregistered to the MPRAGE, then simple subtraction was performed. All quantitative CBF maps were normalized to the MNI space with SPM5. CBF quantification was conducted using an 
ASL data-processing toolbox (23) for NT-ASL and all PPWT-ASLs, which were named as CBF_NT, CBF_Delay_0, CBF_2nd, CBF_Foot, CBF_Peak and CBF_Tail, respectively. In the next step, all CBF files were smoothed with the full-width at half-maximum (FWHM) of the Gaussian smoothing kernel in 6-mm isotropic voxels. All CBFs of the PPWT-ASL were divided by CBF_NT voxel-wise and named as CBF_Delay_0/CBF_NT, $\quad$ CBF_2nd/CBF_NT, $\quad$ CBF_Foot/CBF_NT, CBF_Peak/CBF_NT and CBF_Tail/CBF_NT, to illustrate differences compared with CBF_NT.

We adopted regions of interest (ROIs) based on vascular territory using a 3D stereotactic ROI template (3D-SRT; FUJIFILM RI Pharma, Tokyo, Japan). This software was designed to perform ROI analysis of the brain with anatomical standardization. This method allows ROI analysis of the brain with improved objectivity and excellent reproducibility (24-26). Cerebral regional CBF in the cortico-subcortical regions of each cerebral hemisphere was obtained using the 3D-SRT software. We classified these ROIs into three vascular territories of the anterior cerebral artery (ACA) , anterior part of middle cerebral artery (MCAant) and posterior part of MCA (MCApost), and posterior cerebral artery (PCA), then performed evaluations of these ROIs. 


\section{Statistical analysis}

Repeated measures two-way analysis of variance (ANOVA) with Bonferroni correction was applied for statistical analysis of CBF derived from each ROI of NT-ASL and each cardiac cycle of PPWT-ASL using MedCalc version 12.2.1 software (MedCalc Software, Mariakerke, Belgium). A $P<0.05$ was considered significant. Repeated measures two-way ANOVA with Bonferroni correction was also applied for analysis of the CBF of PPWT-ASL divided by CBF_NT, and a $P<0.05$ was considered significant.

Repeated measures two-way ANOVA with Bonferroni correction was also applied for analysis of Coefficients of variance (CV) of ASL signals of all of the 45 measurements were calculated for NT-ASL and PPTW-ASL. A $P<0.05$ was considered significant.

\section{RESULTS}

Differences in time points of carotid artery flow derived from pre- and post-ASL phase-contrast image among subjects was as follows: $0 \mathrm{~ms}$ for delay $0 \mathrm{~ms}$; 
$25.3 \pm 21.7 \mathrm{~ms}$ for 2 nd point; $50.5 \pm 43.3 \mathrm{~ms}$ for foot; $48.4 \pm 40.4 \mathrm{~ms}$ for peak; and 57.7

$\pm 45.7 \mathrm{~ms}$ for tail.

Mean of CBF images of NT-ASL and PPWT-ASLs of all subjects are shown in Figure 3. Mean Calculated images for CBF of PPWT-ASL divided by CBF_NT of all subjects are shown in Figure .

Results are presented with mean \pm standard error, and $\mathrm{P}$ values according to vascular territory. CBF_NT and CBF of PPWT-ASL are presented in Figure 5. CBFs of PPWT-ASL divided by CBF_NT are presented in Figure 6.

CBF_Foot was significantly lower than CBF_NT, CBF_Delay_0, CBF_Peak and CBF_Tail in ACA, MCAant and MCApost $(\mathrm{P}<0.05) . \mathrm{CBF}$ 2nd was significantly lower than CBF_NT, CBF_Delay_0, CBF_Peak and CBF_Tail except CBF_NT of ACA and CBF_Peak of MCApost $(\mathrm{P}<0.05)$. Other $\mathrm{P}$ values were shown on Table 1 .

CBF_Foot/CBF_NT and CBF_2nd/CBF_NT werer significantly lower than CBF_Delay_0/CBF_NT in ACA and MCAant, and CBF_Foot/CBF_NT was significantly lower than CBF_Peak/CBF_NT in ACA (Table 2).

CVs of NT-ASL and PPWT-ASL were shown on Table 3. Statistical significance was only seen between Delay_0 and 2nd of ACA $(\mathrm{P}=0.0136)$. 


\section{DISCUSSION}

There was a tendency for CBF_Foot to be the lowest, and for CBF_2nd to be the second-lowest $\mathrm{CBF}$ value in ACA and anterior and posterior parts of MCA territories, and our results revealed that CBF_Foot and CBF_2nd were lower than CBF_NT and CBF of the other time points of PPTW-ASL with clear statistical significance for limited ROIs. Wu et al. (16) reported a disparity between ASL signals found between systolic and diastolic tags, and ascribed this to differences in the transit delay of tagged flow. Our results suggest that different CBFs derive from not only systolic tags, but also tags of delay 0 and the tail, suggesting that not only peak carotid systolic flow, but also the subsequent systolic flow (tail) also affects measured CBF changes. From the viewpoint of standardization and feasibleness of PPWT-ASL, time point of Delay 0 might be used routinely since CBF_Delay_0 was relatively high values and less CV. Our results also suggest that exclusion of sampling around 2nd point or foot might lead to increase of CBF values.

CBF_NT turned out to lie approximately within the minimum and maximum CBFs of PPWT-ASL in this study, although statistical significance was seen in several ROIs for several time points of PPWT-ASL. CBF_NT might be assumed to be PPWT-ASL with randomly set triggered time during the scan time, and our results may 
show the characteristics of NT-ASL.

ROIs of the PCA territory showed different results compared with ROIs of the ACA and anterior and posterior parts of MCA territory in this study. No tendencies toward a CBF decrease were seen in diastolic tags such as CBF_2nd and CBF_Foot in the PCA territory. CBF_Peak or CBF_Peak/CBF_NT was higher than the other CBFs. The PCA is known to show a relatively long arterial transit time (27), so a TI2 of 1600 ms was relatively early for CBF quantification in the PCA territory. Residual intravascular tagged flow may play a central role in signal intensity in the PCA territory, leading to relatively higher CBF values for the PCA territory and different patterns for CBF_NT and CBF of the PPTW-ASL in this study. Anatomical variations at the circle of Willis might affect the CBF values of ACA, MCAant, MCApost and PCA (28). Multi-TI experiments are more accurate and immune to the transit time effect, however, it would be more time-consuming, and physiologic state might change and motion artifact might occur during experiments.

In ASL imaging, a gap is inserted between the tagging slab and the imaging slices, due to imperfections in the spatial selectivity of the tag pulses (29). Transit delays thus emerge to allow for the delivery of tags from the tagging region to the imaging plane. Transit delay has been known to vary with location (6) and physiological 
status $(16,18,30)$. Wu et al. (29) concluded that velocity-selective ASL (VS-ASL) with a relatively low velocity cutoff will be suitable for quantification in ASL imaging. Our results show that $\mathrm{CBF}$ _NT was higher than CBF_Foot, probably attributable to the transit delay of tagged blood during the foot of the carotid flow curve. On the other hand, CBF_Delay_0 or CBF_Tail may substitute for VS-ASL with a low-velocity cutoff, although a future comparison study is necessary. Moreover intravoxel vessels are also affected pulsatility since cerebral capillary pulsatility has been observerd in animal study (31).CBF estimation in ASL is based upon the assumption that labeled blood does not contribute to signal, it is impossible to exclude the effect of capillary pulsatility on CBF estimation.

Significant maximum values of CBF for PPWT-ASL during the cardiac cycle were not identified in this study. If a maximum CBF for PPWT-ASL exists, the difference between maximum and minimum CBFs of PPWT-ASL could reflect vascular resistance or vascular reserve. We sampled only 5 time points of PPWT-ASL, and certain sampling errors and problems with the relevance of the sampling points may exist. Although PPWT-ASL with more sampling time points may offer a better approach to evaluating the effects of cardiac cycle on CBF, longer imaging time, motion artifacts, and fluctuations in the physiological status of subjects will remain controversial issues. 
The duration of labeling time may have influenced our results for CBF with PPWT-ASL. A number of thin slice sinc saturation pulse was applied at the distal end of tagging region from TI1 until TIs in Q2TIPS (6), which assures the amount of tagged blood volume until TI1. TI1 was fixed at $700 \mathrm{~ms}$ in this study, and $700 \mathrm{~ms}$ of TI1 will be too long for subjects with relative tachycardia, and too short for those with bradycardia. The proportionate of TI1 compared to PR may lead to equal distribution of PPWT-CBF, however, which would increase the signal from ASL itself. A very short duration of TI1 (e.g., $100 \mathrm{~ms}$ ) would provide an equally proportional TI1, but the signal gain would be inadequate for analysis (19).

Tagging efficeinecy is an important factor in estimation of CBF on ASL. CASL and PCASL are relatively vulnerable to tagging insufficiency due to multiple tagging pulse before readout, and the experimental data showed a significant correlation between labeling efficiency and flow velocity (32) . On the contrary, a 10 ms adiabatic inversion pulses is applied as a selective saturation which covers $10 \mathrm{~cm}$ width below the imaging slab in PASL, tagging efficiency of PASL is less impaired than that of PCASL, and experimentally calculated tagging efficiency turned out to be between 0.976 and 0.978 even under flow velocity of $100 \mathrm{~cm} / \mathrm{sec}(33)$. Arterial arrival time $(\Delta \mathrm{t})$ also changes during cardiac cycle and affects value of CBF in consideration of pulsatile 
parabolic arterial flow (34).

Several limitations must be considered in this study. ECG-triggered ASL is more accurate than PPWT-ASL, at least in principle. A longer T1 of blood and tissue at a higher magnetic field offers a better signal to noise ratio (SNR) for ASL (19), but the magnetohydrodynamic effect may superimpose artifacts onto the ECG signal (35). This effect generates voltages related to blood flow through a magnetic field, which may lead to incorrect R-wave detection. Although simultaneous recording of pulse waves was unavailable in this study, differences in time points between pre- and post-ASL phase-contrast imaging were restricted but not neglibile. In other words, the time point of Delay 0 was consistent and easily applied to clinical settings. On the other hand, the other time points of longer delay time might be less consistent and scan time will be long.

Effective TR was variable under PPWT-ASL, representing another limitation. TR is no longer a constant for each acquisition in PPWT-ASL. Inconsistency of spin history under inconsistent TR may lead to ASL signal fluctuations. The effective TR is 3000 ms for NT-ASL, and more than 3000 for PPWT-ASL. Since sufficient time was left for the saturated blood to pass through, this study ensured the consistency of both spin history and ASL signal regardless of TR variations. Effective TR became longer in 
proportionate to delay of trigger time points, however, CBF of PPTW-ASL seems less affected to the delay of trigger time points. In addition, effective TR varies depending on pulse rate and it is impossible to evaluate its impact on CBF of PPTW-ASL of each subject.

In conclusion, PPWT-ASL strongly affects CBF values compared with NT-ASL, particularly with the foot of the carotid artery flow, since CBF_Foot and CBF_2nd were lower than CBF_NT and CBF at other time points for PPWT-ASL in the ACA and MCA territories, with clear statistical significance. CBF_NT was assumed to lie approximately between minimum and maximum $\mathrm{CBF}$ with clear statistical significance in several ROIs at several time points of PPWT-ASL. 


\section{References}

1. Williams DS, Detre JA, Leigh JS, Koretsky AP. Magnetic resonance imaging of perfusion using spin inversion of arterial water. Proc Natl Acad Sci U S A 1992;89(1):212-216.

2. Edelman RR, Siewert B, Darby DG, Thangaraj V, Nobre AC, Mesulam MM, Warach S. Qualitative mapping of cerebral blood flow and functional localization with echo-planar MR imaging and signal targeting with alternating radio frequency. Radiology 1994;192(2):513-520.

3. Kim SG. Quantification of relative cerebral blood flow change by flow-sensitive alternating inversion recovery (FAIR) technique: application to functional mapping. Magn Reson Med 1995;34(3):293-301.

4. Kwong KK, Chesler DA, Weisskoff RM, Donahue KM, Davis TL, Ostergaard L, Campbell TA, Rosen BR. MR perfusion studies with T1-weighted echo planar imaging. Magn Reson Med 1995;34(6):878-887.

5. Alsop DC, Detre JA. Reduced transit-time sensitivity in noninvasive magnetic resonance imaging of human cerebral blood flow. J Cereb Blood Flow Metab 1996;16(6):1236-1249.

6. Wong EC, Buxton RB, Frank LR. Quantitative imaging of perfusion using a single subtraction (QUIPSS and QUIPSS II). Magn Reson Med 1998;39(5):702-708.

7. Noguchi T, Kawashima M, Irie H, Ootsuka T, Nishihara M, Matsushima T, Kudo S. Arterial spin-labeling MR imaging in moyamoya disease compared with SPECT imaging. Eur J Radiol 2011;80(3):e557-562.

8. Alsop DC, Detre JA. Multisection cerebral blood flow MR imaging with continuous arterial spin labeling. Radiology 1998;208(2):410-416.

9. Kimura H, Kado H, Koshimoto Y, Tsuchida T, Yonekura Y, Itoh H. Multislice continuous arterial spin-labeled perfusion MRI in patients with chronic occlusive cerebrovascular disease: a correlative study with CO2 PET validation. J Magn Reson Imaging 2005;22(2):189-198.

10. Wong EC. Vessel-encoded arterial spin-labeling using pseudocontinuous tagging. Magn Reson Med 2007;58(6):1086-1091.

11. Bokkers RP, Bremmer JP, van Berckel BN, Lammertsma AA, Hendrikse J, Pluim JP, Kappelle LJ, Boellaard R, Klijn CJ. Arterial spin labeling perfusion MRI at multiple delay times: a correlative study with $\mathrm{H}(2)(15) \mathrm{O}$ positron emission tomography in 
patients with symptomatic carotid artery occlusion. J Cereb Blood Flow Metab 2010;30(1):222-229.

12. Gunther M, Oshio K, Feinberg DA. Single-shot 3D imaging techniques improve arterial spin labeling perfusion measurements. Magn Reson Med 2005;54(2):491-498.

13. Fushimi Y, Okada T, Yamamoto A, Kanagaki M, Imai H, Togashi K. Estimation of the timing of carotid artery flow using peripheral pulse wave-gated MRI. J Magn Reson Imaging 2012;36(2):454-458.

14. Holdsworth DW, Norley CJ, Frayne R, Steinman DA, Rutt BK. Characterization of common carotid artery blood-flow waveforms in normal human subjects. Physiol Meas 1999;20(3):219-240.

15. Hoi Y, Wasserman BA, Xie YJ, Najjar SS, Ferruci L, Lakatta EG, Gerstenblith G, Steinman DA. Characterization of volumetric flow rate waveforms at the carotid bifurcations of older adults. Physiol Meas 2010;31(3):291-302.

16. Wu WC, Mazaheri Y, Wong EC. The effects of flow dispersion and cardiac pulsation in arterial spin labeling. IEEE Trans Med Imaging 2007;26(1):84-92.

17. Wu WC, Edlow BL, Elliot MA, Wang J, Detre JA. Physiological modulations in arterial spin labeling perfusion magnetic resonance imaging. IEEE Trans Med Imaging 2009;28(5):703-709.

18. Buxton RB, Frank LR, Wong EC, Siewert B, Warach S, Edelman RR. A general kinetic model for quantitative perfusion imaging with arterial spin labeling. Magn Reson Med 1998;40(3):383-396.

19. Luh WM, Wong EC, Bandettini PA, Hyde JS. QUIPSS II with thin-slice TI1 periodic saturation: a method for improving accuracy of quantitative perfusion imaging using pulsed arterial spin labeling. Magn Reson Med 1999;41(6):1246-1254.

20. Silva AC, Kim SG, Garwood M. Imaging blood flow in brain tumors using arterial spin labeling. Magn Reson Med 2000;44(2):169-173.

21. Wang J, Aguirre GK, Kimberg DY, Roc AC, Li L, Detre JA. Arterial spin labeling perfusion fMRI with very low task frequency. Magn Reson Med 2003;49(5):796-802.

22. Lu H, Clingman C, Golay X, van Zijl PC. Determining the longitudinal relaxation time (T1) of blood at 3.0 Tesla. Magn Reson Med 2004;52(3):679-682.

23. Wang Z, Aguirre GK, Rao H, Wang J, Fernandez-Seara MA, Childress AR, Detre JA. Empirical optimization of ASL data analysis using an ASL data processing toolbox: ASLtbx. Magn Reson Imaging 2008;26(2):261-269.

24. Takeuchi R, Yonekura Y, Takeda SK, Fujita K, Konishi J. Fully automated quantification of regional cerebral blood flow with three-dimensional stereotaxic 
region of interest template: validation using magnetic resonance imaging--technical note. Neurol Med Chir (Tokyo) 2003;43(3):153-162.

25. Takeuchi R, Sengoku T, Matsumura K. Usefulness of fully automated constant ROI analysis software for the brain: 3DSRT and FineSRT. Radiat Med 2006;24(7):538-544.

26. Tateno M, Utsumi K, Kobayashi S, Takahashi A, Saitoh M, Morii H, Fujii K, Teraoka M. Usefulness of a blood flow analyzing program 3DSRT to detect occipital hypoperfusion in dementia with Lewy bodies. Prog Neuropsychopharmacol Biol Psychiatry 2008;32(5):1206-1209.

27. Robson PM, Dai W, Shankaranarayanan A, Rofsky NM, Alsop DC. Time-resolved Vessel-selective Digital Subtraction MR Angiography of the Cerebral Vasculature with Arterial Spin Labeling. Radiology 2010;257(2):507-515.

28. Hendrikse J, van Raamt AF, van der Graaf Y, Mali WP, van der Grond J. Distribution of cerebral blood flow in the circle of Willis. Radiology 2005;235(1):184-189.

29. Wu WC, Wong EC. Intravascular effect in velocity-selective arterial spin labeling: the choice of inflow time and cutoff velocity. Neuroimage 2006;32(1):122-128.

30. Yang Y, Engelien W, Xu S, Gu H, Silbersweig DA, Stern E. Transit time, trailing time, and cerebral blood flow during brain activation: measurement using multislice, pulsed spin-labeling perfusion imaging. Magn Reson Med 2000;44(5):680-685.

31. Rashid S, McAllister JP, 2nd, Yu Y, Wagshul ME. Neocortical capillary flow pulsatility is not elevated in experimental communicating hydrocephalus. J Cereb Blood Flow Metab 2012;32(2):318-329.

32. Aslan S, Xu F, Wang PL, Uh J, Yezhuvath US, van Osch M, Lu H. Estimation of labeling efficiency in pseudocontinuous arterial spin labeling. Magn Reson Med 2010;63(3):765-771.

33. Wong EC, Buxton RB, Frank LR. A theoretical and experimental comparison of continuous and pulsed arterial spin labeling techniques for quantitative perfusion imaging. Magn Reson Med 1998;40(3):348-355.

34. Gallichan D, Jezzard P. Modeling the effects of dispersion and pulsatility of blood flow in pulsed arterial spin labeling. Magn Reson Med 2008;60(1):53-63.

35. Abi-Abdallah D, Robin V, Drochon A, Fokapu O. Alterations in human ECG due to the MagnetoHydroDynamic effect: a method for accurate $\mathrm{R}$ peak detection in the presence of high MHD artifacts. Conf Proc IEEE Eng Med Biol Soc 2007;2007:1842-1845. 


\section{Captions for Illustrations}

Figure 1

(A) Labeling slab of ASL is shown in solid rectangule and imaging slab is shown in gray filled rectangle. Phase-contrast imaging at the level of the right common carotid artery $1.5 \mathrm{~cm}$ proximal to the carotid bifurcation (dashed line rectangule) was conducted for all subjects, both before and after ASL. (B) Each of the following time points of the carotid pulse wave was visualized on the representative one cardiac cycle data from phase-contrast MR imaging: delay $0 \mathrm{~ms}$ (a); 2nd point (midpoint between delay $0 \mathrm{~ms}$ and foot) (b); foot (c); peak (d); and tail (e).

Figure 2

Representative ROIs for regional CBF in the cortico-subcortical regions of each cerebral hemisphere were obtained using 3D-SRT software. We classified these ROIs into three vascular territories of the ACA (callosomarginal and pericallosal ROIs), the anterior part of MCA (precentral and central ROIs), the posterior part of MCA (parietal and angular ROIs), and PCA (occipital and thalamus ROIs). 
Figure 3

Mean images of CBF_NT and CBF for each cardiac cycle of PPWT-ASL of all subjects. Differences among CBF_NT and CBF_PPWT-ASL are relatively mild, but obvious changes are difficult to detect. Color calibration bar $=\mathrm{ml} / 100 \mathrm{~g} / \mathrm{min}$.

Figure 4

Mean calculated images of CBF for PPWT-ASL divided by CBF_NT of all subjects. Pixels larger than CBF_NT are shown as red color images (scale between 1.00 and 1.25), and pixels smaller than CBF_NT are shown as color images in the blue color image (scale between 0.75 and 1.00). Note that pixels smaller than CBF_NT are very prominent in the ACA and MCA territories of CBF_Foot/CBF_NT. Pixels larger than CBF_NT are relatively prominent in the PCA territories of CBF_Peak/CBF_NT. Pixel values of larger than 5 were removed as outliers during division and average process.

Figure 5

CBF_NT and CBF of PPWT-ASL are shown in this graph. CBF_Foot was the lowest and CBF_2nd was the second lowest in the ACA, MCAant MCApost ROIs. CBF_NT lay approximately between CBF_NT and CBF of PPWT-ASL in the ACA and 
MCA territories. CBF_Peak was the highest in the PCA ROI.

Figure 6

Voxel-wise calculations for CBF of PPWT-ASL divided by CBF_NT. CBF_Foot/CBF_NT was the lowest and CBF_2nd/CBF_NT was the second-lowest in the ROIs of the ACA, MCAant and MCApost ROIs. CBF_Peak/CBF_NT was the highest in the PCA ROI. 


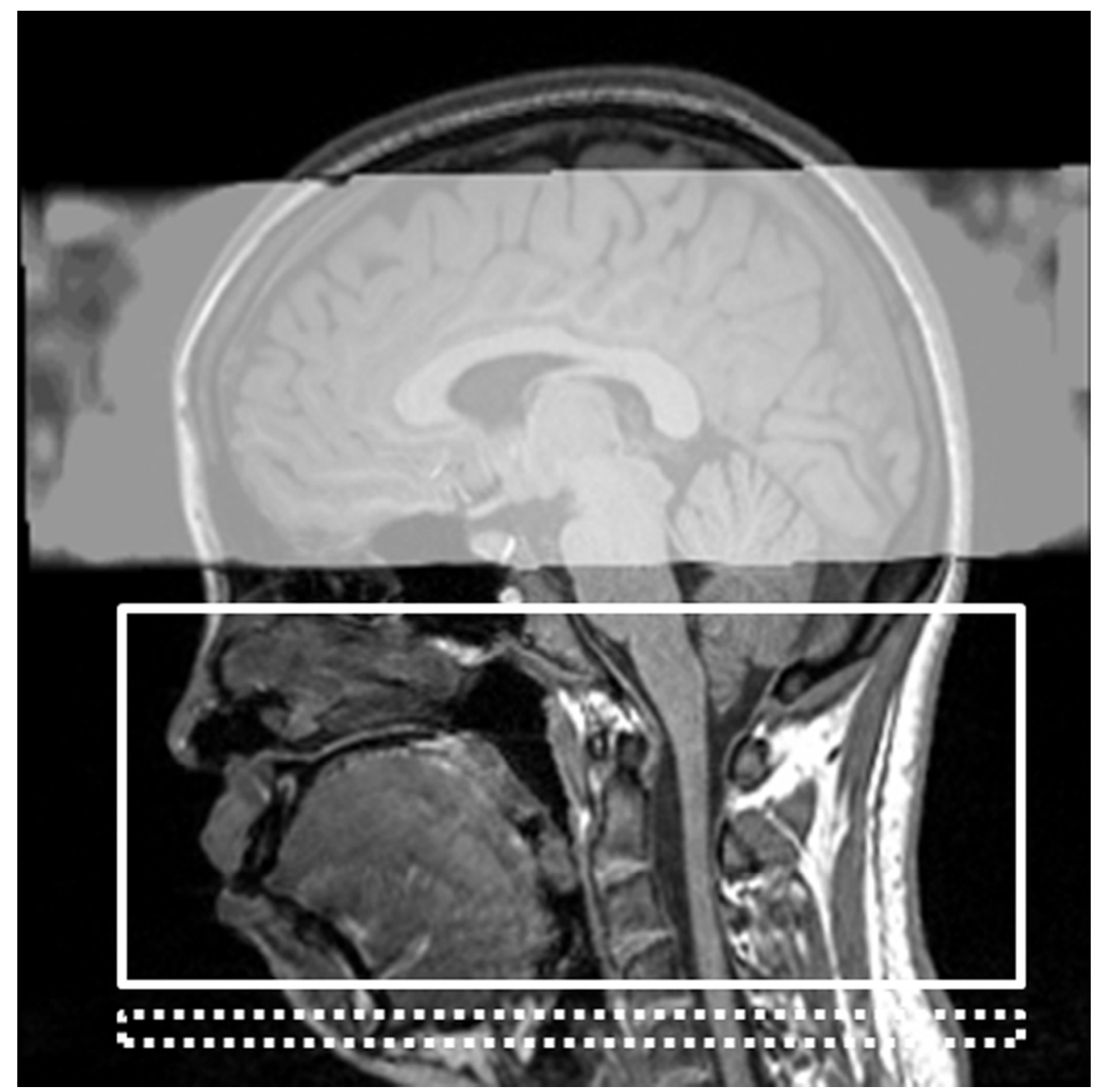

Figure 1

(A) Labeling slab of ASL is shown in solid rectangule and imaging slab is shown in gray filled rectangle. Phase-contrast imaging at the level of the right common carotid artery $1.5 \mathrm{~cm}$ proximal to the carotid bifurcation (dashed line rectangule) was conducted for all subjects, both before and after ASL. (B) Each of the following time points of the carotid pulse wave was visualized on the representative one cardiac cycle data from phase-contrast MR imaging: delay $0 \mathrm{~ms}(\mathrm{a})$; 2nd point (midpoint between delay $0 \mathrm{~ms}$ and foot) (b); foot (c); peak (d); and tail (e).

$72 \times 72 \mathrm{~mm}(300 \times 300 \mathrm{DPI})$ 
Figure 1

(A) Labeling slab of ASL is shown in solid rectangule and imaging slab is shown in gray filled rectangle. Phase-contrast imaging at the level of the right common carotid artery $1.5 \mathrm{~cm}$ proximal to the carotid bifurcation (dashed line rectangule) was conducted for all subjects, both before and after ASL. (B) Each of the following time points of the carotid pulse wave was visualized on the representative one cardiac cycle data from phase-contrast MR imaging: delay $0 \mathrm{~ms}(\mathrm{a})$; 2 nd point (midpoint between delay $0 \mathrm{~ms}$ and foot)

(b); foot (c); peak (d); and tail (e).

$99 \times 67 \mathrm{~mm}(300 \times 300$ DPI $)$ 


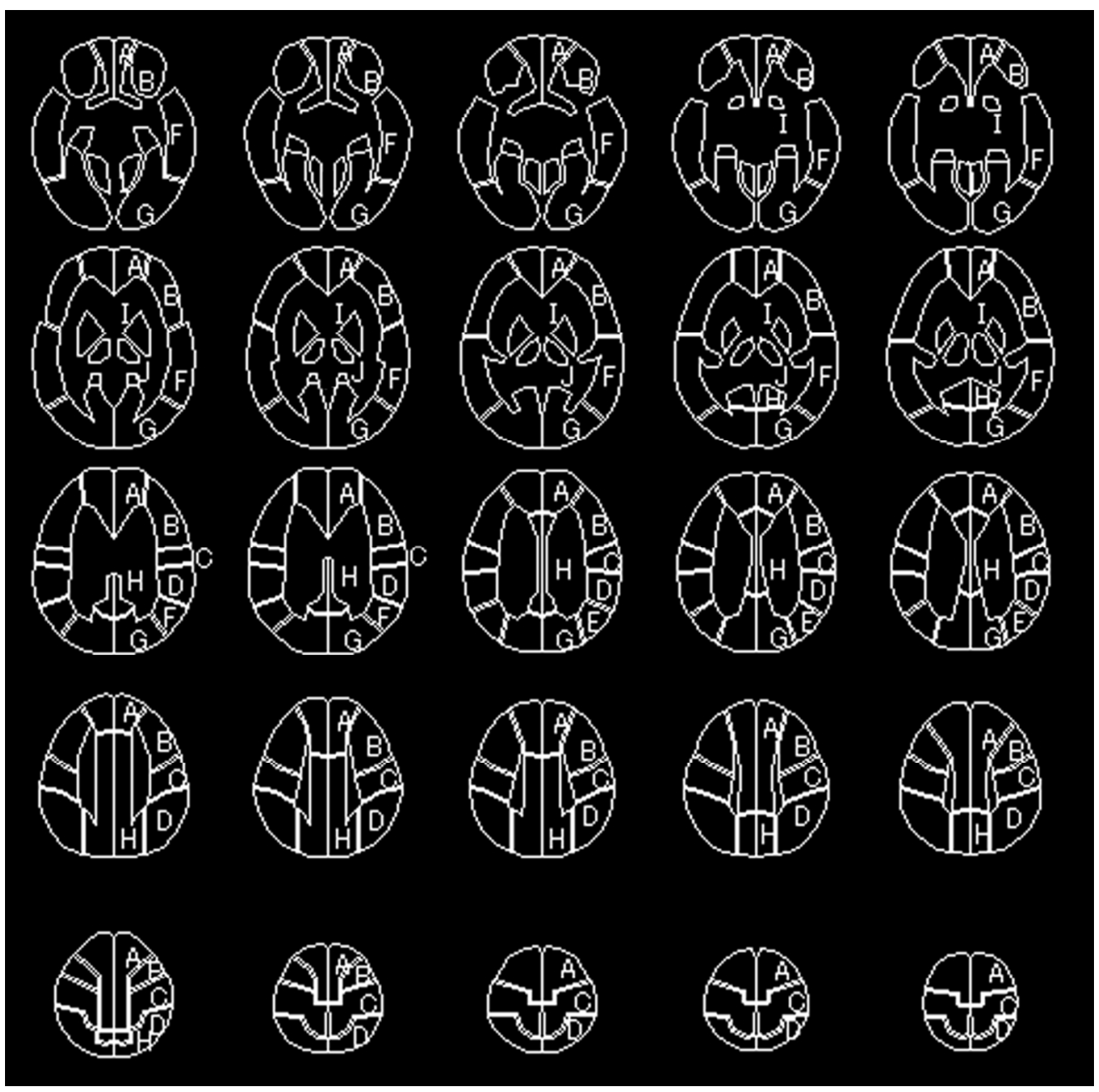

Figure 2

Representative ROIs for regional CBF in the cortico-subcortical regions of each cerebral hemisphere were obtained using 3D-SRT software. We classified these ROIs into three vascular territories of the ACA (callosomarginal and pericallosal ROIs), the anterior part of MCA (precentral and central ROIs), the posterior part of MCA (parietal and angular ROIs), and PCA (occipital and thalamus ROIs).

\section{$84 \times 83 \mathrm{~mm}(300 \times 300 \mathrm{DPI})$}




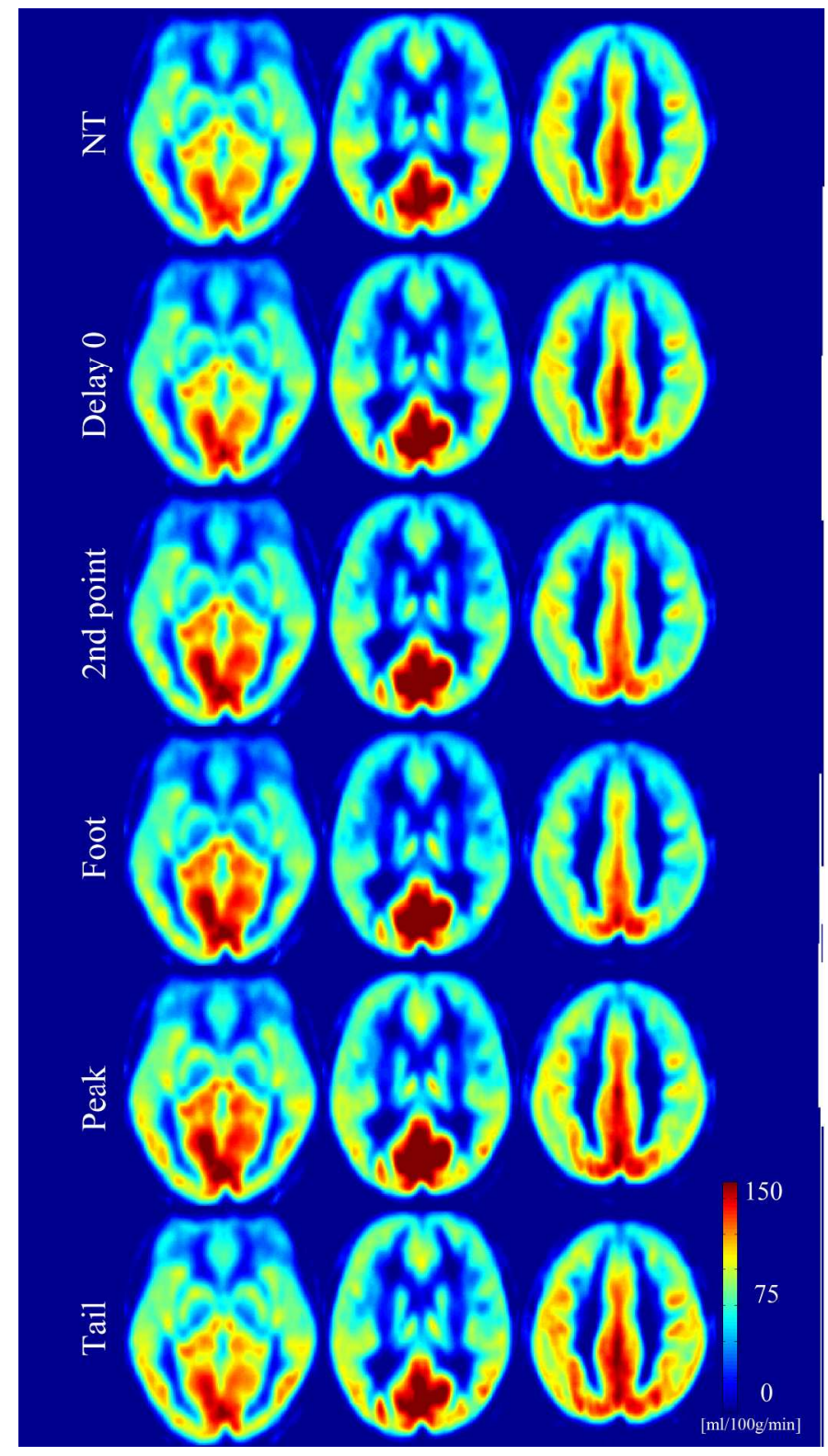

Figure 3

Mean images of CBF_NT and CBF for each cardiac cycle of PPWT-ASL of all subjects. Differences among CBF_NT and CBF_PPWT-ASL are relatively mild, but obvious changes are difficult to detect. Color calibration bar $=\mathrm{ml} / 100 \mathrm{~g} / \mathrm{min}$. 


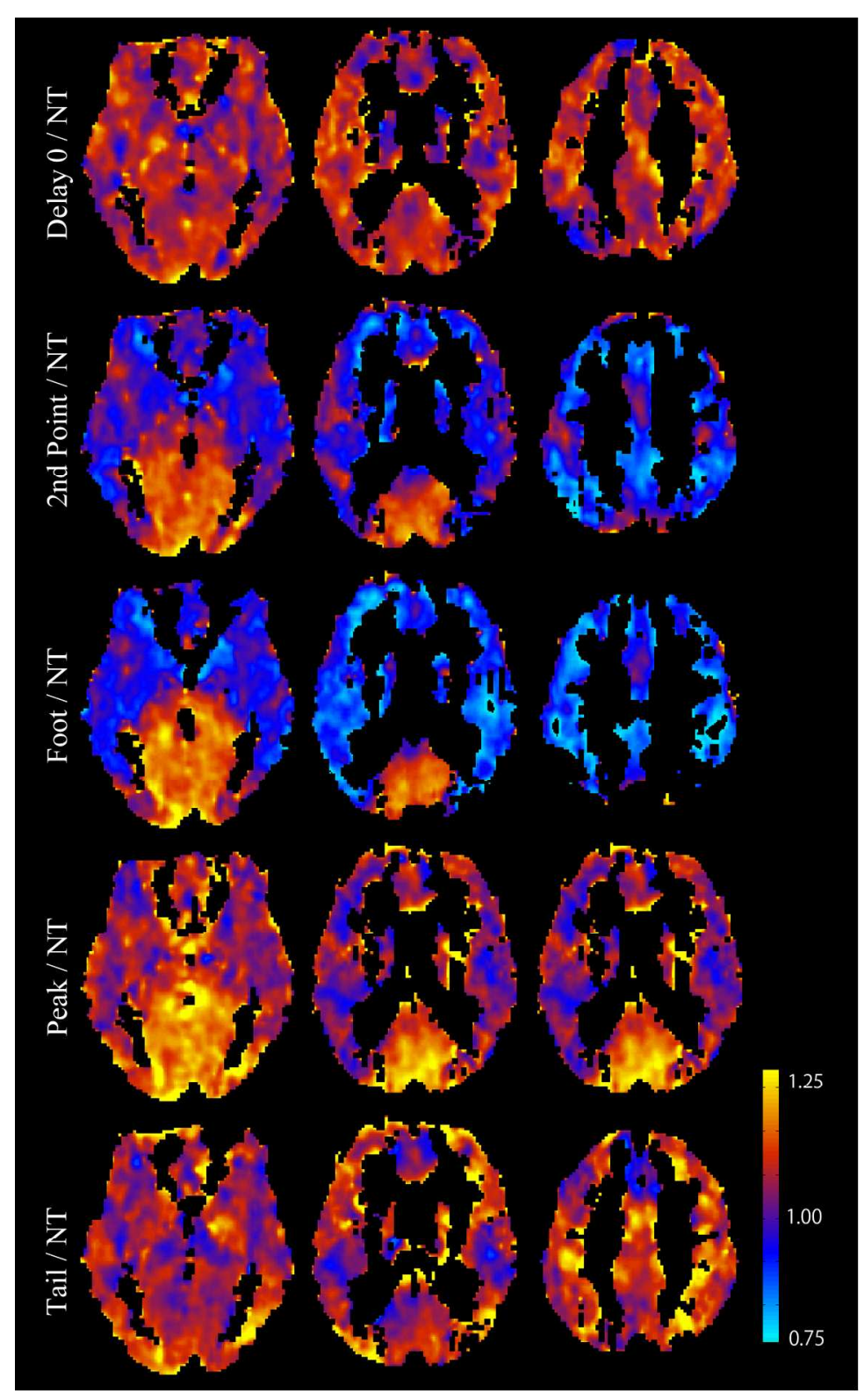

Figure 4.

Mean calculated images of CBF for PPWT-ASL divided by CBF_NT of all subjects. Pixels larger than CBF_NT are shown as red color images (scale between 1.00 and 1.25), and pixels smaller than CBF_NT are shown as color images in the blue color image (scale between 0.75 and 1.00 ). Note that pixels smaller than CBF_NT are very prominent in the ACA and MCA territories of CBF_Foot/CBF_NT. Pixels larger than CBF_NT are relatively prominent in the PCA territories of CBF_Peak/CBF_NT. Pixel values of larger than 5 were removed as outliers during division and average process.

$$
235 \times 383 \mathrm{~mm}(300 \times 300 \mathrm{DPI})
$$




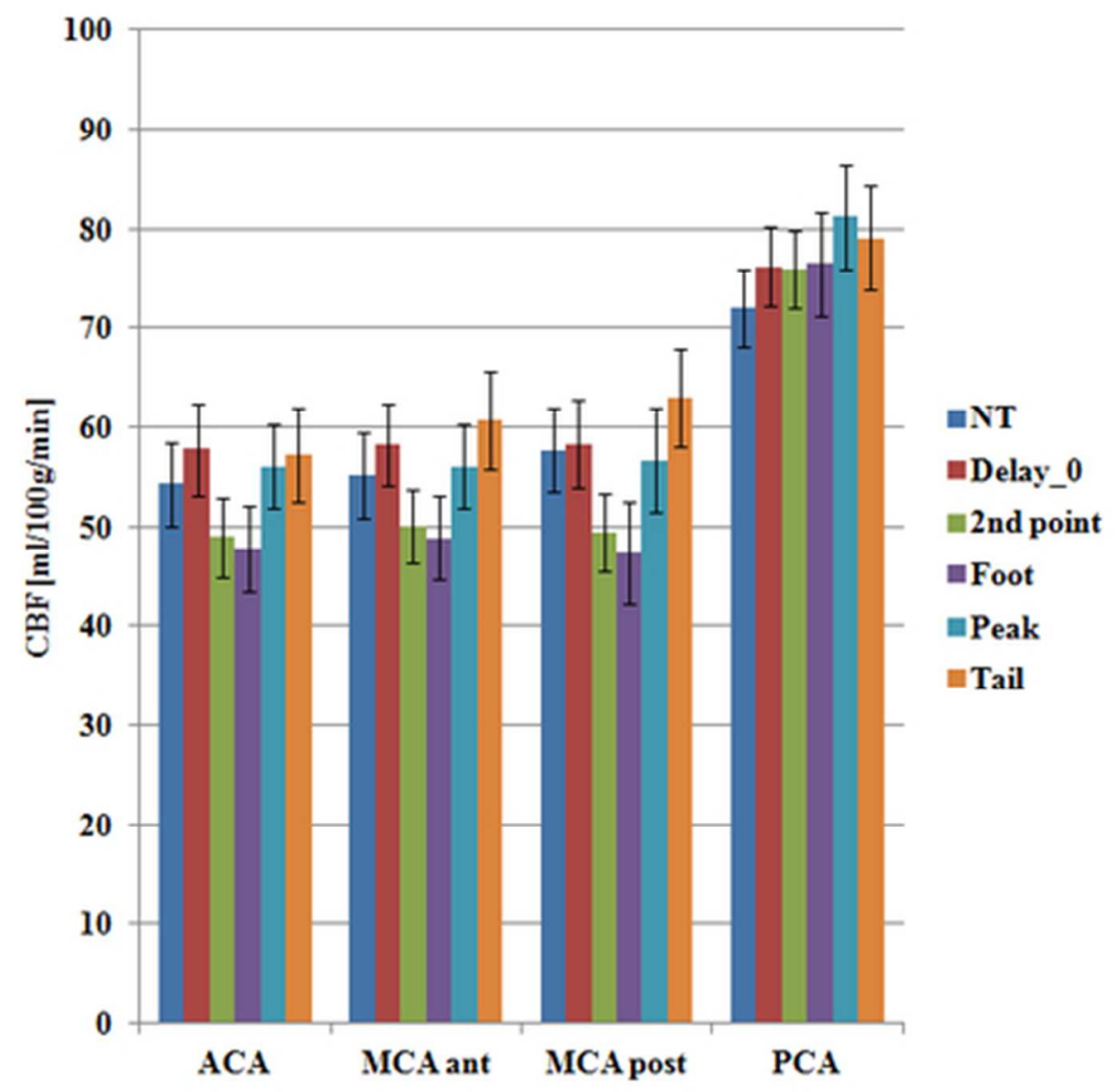

Figure 5.

CBF_NT and CBF of PPWT-ASL are shown in this graph. CBF_Foot was the lowest and CBF_2nd was the second lowest in the ACA, MCAant MCApost ROIs. CBF_NT lay approximately between CBF_NT and CBF of PPWT-ASL in the ACA and MCA territories. CBF_Peak was the highest in the PCA ROI.

$39 \times 37 \mathrm{~mm}(300 \times 300 \mathrm{DPI})$ 


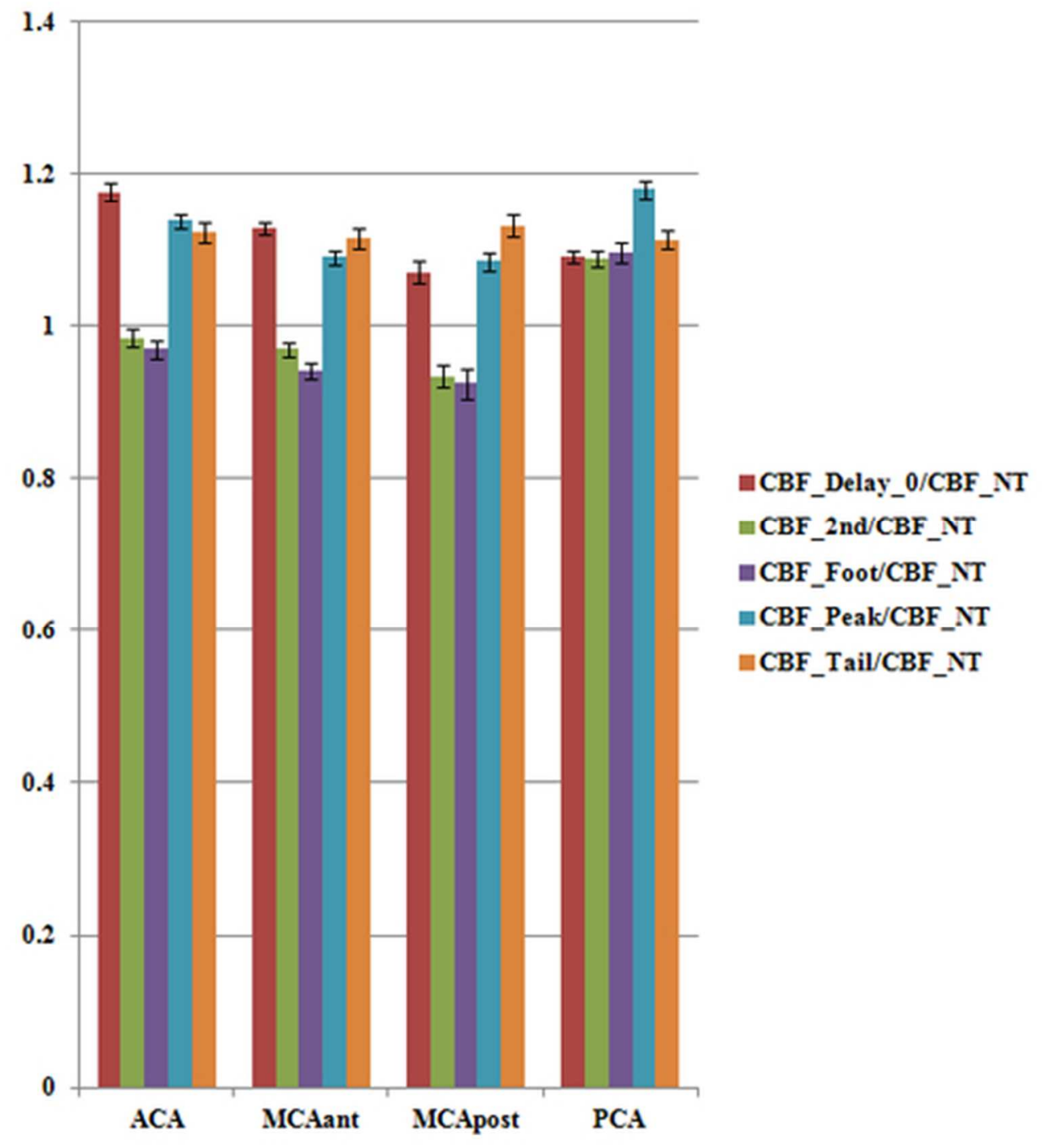

Figure 6.

Voxel-wise calculations for CBF of PPWT-ASL divided by CBF_NT. CBF_Foot/CBF_NT was the lowest and CBF_2nd/CBF_NT was the second-lowest in the ROIs of the ACA, MCAant and MCApost ROIs. CBF_Peak/CBF_NT was the highest in the PCA ROI.

$$
42 \times 46 \mathrm{~mm} \text { (300 x } 300 \text { DPI) }
$$


Table 1. Results of statistical analysis among CBF of NT-ASL and PPWT-ASL

\begin{tabular}{|c|c|c|c|c|c|}
\hline$P$ value & & ACA & MCAant & MCApost & PCA \\
\hline CBF_NT & vs. CBF_Dela! & 0.181 & 0.0676 & 1 & $0.0345^{*}$ \\
\hline & vs. CBF_2nd & 0.0518 & $0.0249 *$ & $0.0136^{*}$ & 0.2285 \\
\hline & vs. CBF_Foot & $0.0107 *$ & $0.0073 *$ & $0.007 *$ & 0.6984 \\
\hline & vs. CBF_Peak & 1 & 1 & 1 & $0.0002 *$ \\
\hline & vs. CBF_Tail & 0.2644 & $0.0051 *$ & $0.0174 *$ & $0.007 *$ \\
\hline CBF_Dela】 & vs. CBF_2nd & $0.0001 *$ & $<0.0001 *$ & $0.0047^{*}$ & 1 \\
\hline & vs. CBF_Foot & $<0.0001 *$ & $0.0001 *$ & $0.0022 *$ & 1 \\
\hline & vs. CBF_Peak & 1 & 1 & 1 & $0.0365 *$ \\
\hline & vs. CBF_Tail & 1 & 1 & 0.1409 & 1 \\
\hline CBF_2nd & vs. CBF_Foot & 1 & 1 & 1 & 1 \\
\hline & vs. CBF_Peak & $0.0004 *$ & $0.012 *$ & 0.0592 & $0.0229 *$ \\
\hline & vs. CBF_Tail & $0.0008 *$ & $<0.0001 *$ & $0.0002^{*}$ & 0.8143 \\
\hline CBF_Foot & vs. CBF_Peak & $<0.0001 *$ & $0.0002 *$ & $0.014^{*}$ & $0.0143 *$ \\
\hline & vs. CBF_Tail & $0.0002 *$ & $<0.0001^{*}$ & $<0.0001 *$ & 1 \\
\hline CBF_Peak & vs. CBF_Tail & 1 & 0.0747 & $0.0106^{*}$ & 1 \\
\hline
\end{tabular}


5 Table 2. Results of statistical analysis among CBF of PPWT-ASL divided by CBF_NT 7

CBF_Delay_0/CBF_NT vs. CBF_2nd/CBF_NT

ACA

MCAant

MCApost

PCA

11
12

13

14

15

16

17

18

19

20

21

22

23

24

25

26

27

28

29

30

31

32

33

34

35

36

37

38

39

40

41

42

43

44

45

46

47

CBF_2nd/CBF_NT vs. CBF_Foot/CBF_NT

vs. CBF_Peak/CBF_NT

$0.0095^{*}$

$0.0262^{*}$

0.4382

1

vs. $\mathrm{CBF}_{-}$Peak/CBF_NT

0.442

0.3404

vs. CBF_Tail/CBF_NT

1

1

1

CBF_Foot/CBF_NT vs. CBF_Peak/CBF_NT

$0.0111 *$

1
0.3303

1
0.2424

0.0508

.2424

0.1387

vs. CBF_Tail/CBF_NT

0.3428

0.2237

1

vs. CBF_Tail/CBF_NT

0.5619

0.243

0.3163

0.0648

CBF_Peak/CBF_NT vs. CBF_Tail/CBF_NT

1

1

0.2781

1

\begin{tabular}{l}
48 \\
\hline
\end{tabular} 
Table 3. CVs of NT-ASL and PPWT-ASL. 